Case Reports

\title{
Upper Extremity Superficial Vein Thromboses Presenting as Acute Neck Pain in a Young and Healthy Male: A Case Report
}

Kristen L Zosel, PT, DPT ${ }^{1}$, Max K Dummar, PT, DPT ${ }^{2}$, Benjamin G Adams, PT, DPT ${ }^{1}$, Nancy C Henderson, PT, DPT, PhD

${ }^{3}$, Richard B Westrick, PT, DPT, DSc ${ }^{1}$

${ }^{1}$ U.S. Army Research Institute of Environmental Medicine, ${ }^{2}$ McDonald Army Health Center, U.S. Army, ${ }^{3}$ Georgia Southern University

Keywords: upper extremity, superficial vein thrombosis, neck pain

https://doi.org/10.26603/001c.23429

\section{International Journal of Sports Physical Therapy}

Vol. 16, Issue 3, 2021

\section{Background and Purpose}

Neck pain in the United States is pervasive and contributes to disability. While the majority of neck pain in young and healthy individuals is neuromusculoskeletal in nature, screening for red flags is necessary for ruling-out serious medical pathologies. The purpose of this case report is to describe a young and healthy male subject with a primary complaint of acute neck pain with multiple underlying upper extremity superficial vein thromboses (UESVTs).

\section{Case Description}

The subject was a 27-year-old male active-duty Soldier referred to physical therapy by his primary care provider (PCP) for acute left-sided neck pain. Prior to physical therapy, the subject had been treated with cyclobenzaprine, oxycodone-acetaminophen, trigger point injection and had undergone a D-dimer to rule out a potential thrombus due to air travel and lower extremity immobilization.

\section{Outcomes}

The subject underwent a D-dimer, Doppler ultrasound, pharmacological treatment of Rivaroxaban, and was referred to hematology/oncology to rule out systemic causes of SVTs. Evidence of subtle increases in blood pressure over the course of three months, a positive D-dimer, and symptoms incongruent with clinical presentation contributed to referral to a hematology/oncology specialist and a diagnosis of multiple UESVTs. The subject was able to return to his previous level of activity by six months and remained free of SVTs at two-year follow-up.

\section{Discussion}

UESVT events are rare and can be challenging to identify. This case report describes a unique presentation of acute neck pain caused by underlying UESVTs in an otherwise healthy and active young male.

\section{Level of Evidence}

Level 4

\section{BACKGROUND AND PURPOSE}

Young and healthy subjects presenting with neck pain in the absence of a clear mechanism of injury or apparent con- tributing factors can present unique challenges. Determining if the subject is appropriate for physical therapy care can be especially challenging when the subject is referred to physical therapy from a physician assistant following a visit

\footnotetext{
a Corresponding author: Kristen L. Zosel

U.S. Army Research Institute of Environmental Medicine Natick, MA, 01760, United States

Telephone: 210-406-0029

email: kristen.l.wilburn.ctr@mail.mil
} 
to the emergency department. While neck pain is a common occurrence, affecting $15.1 \%$ of the U.S. population during any given three-month period, ${ }^{1,2}$ the potential for serious underlying conditions requiring referral exist. Screening for red flags in subjects with neck pain is critical to the clinical decision-making process and determination of appropriateness for physical therapy services.

The development of UESVTs are largely restricted to hospitalized populations; use of endovascular devices (e.g. PICC lines and peripheral venous lines) and in subjects with malignant cancers, infection or inflammatory processes, during pregnancy/post-partum or with haptogastrointestinal pathologies. ${ }^{3,4}$ Much less commonly (incidence 1/ $100,000)$, UESVTs have been reported in male subjects under the age of 30 due to Paget-Schroetter Syndrome (effort thrombosis) or Thoracic Outlet Syndrome (TOS). ${ }^{4,5}$ In these rare cases, the presence of repetitive upper extremity movements cause microtrauma to the endothelium creating thromboses. Clinical signs of UESVTs occur in roughly a third of those diagnosed, with the most common signs including erythema, indurated cord, edema, and pain. ${ }^{4,6}$

The purpose of this case report is to describe an example of a young and healthy subject presenting with a primary complaint of neck pain with underlying UESVTs.

\section{CASE DESCRIPTION}

The subject was a 27-year-old, right-hand dominant, male, active-duty U.S. Army Soldier with a chief complaint of acute 12-day history of left-sided neck pain (Figure 1). The subject's neck pain was preceded by a relatively short, twohour airplane flight, in which he reported falling asleep in an "awkward position" for approximately 20 minutes. Following the flight, the subject initially reported experiencing neck stiffness which progressed to mild pain by the next morning, and to moderate pain by day 8 , prompting the subject to schedule an initial encounter for neck pain with his PCP. The subject denied significant past medical history or recent events of trauma to his neck but did report having awoken with similar neck pain about three months prior which resolved on it's on within the same week. The subject was in excellent overall health with the exception of a healing Lisfranc injury to his left lower extremity which he incurred two months prior and had used bilateral axillary crutches for approximately six weeks after the lower extremity injury. The timeline of encounters is represented in Figure 1.

Treatment for this episode of neck pain included the subject visiting a civilian chiropractor twice and a massage therapist once before seeing his PCP for neck pain. The subject reported minimal temporary relief of his symptoms and growing difficulty in being able to maintain any prolonged positions with his visits to the chiropractor and massage therapist. Nine days following onset of pain, and the subject's first visit with his PCP, he was treated with trigger point injections (Marcaine/Lidocaine) in the upper trapezius muscle with temporary relief in symptoms. His PCP also prescribed Hydrocodone/Acetaminophen (5mg/325mg), Ibuprofen (800mg), and Cyclobenzaprine (10 mg).

On day 11 following initial onset of symptoms, the subject was unable to sleep or find any relief with medication or changing positions and visited a civilian emergency department. The emergency room physician ordered cervical spine radiographs which were unremarkable and diagnosed him with a neck strain. He was prescribed Percocet for pain relief and instructed to follow-up with his PCP. The next day he followed-up with his PCP (PCP visit number 2, 12 days since the onset of his pain) during which a D-dimer test was ordered and he was referred to physical therapy. The subject's PCP requested he be seen as soon as possible for acute neck pain. The subject's most immediate goal was to manage pain so that he could get some sleep.

\section{INITIAL PHYSICAL THERAPY EVALUATION}

The subject described his neck symptoms as constant, variable, sharp, deep, and primarily located over his mid- to lower-left cervical paraspinal musculature with some discomfort noted in the left upper trapezius and scapular region. He denied radiating pain into either upper extremity, numbness or tingling, double vision, blurry vision, difficulty swallowing, dizziness, upper extremity weakness, or unexpectedly dropping objects. The subject had no observable dysarthria, nystagmus or ataxia of gait. He rated his pain with medication (Percocet) at a $2 / 10$ on the Numeric Pain Rating Scale (NPRS) ${ }^{7}$ during the evaluation, with complaints of neck stiffness, particularly during cervical extension. He reported that his pain increased to $10 / 10$ on the NPRS with active movement, especially looking up, in addition to significant difficulty laying down in any position to sleep.

Observation of the subject revealed no erythema, edema, or deformity of the neck or upper extremities. The subject's active range of motion was restricted in cervical extension, and he was unable to position himself in neutral due to pain. Cervical flexion was limited to 10 degrees before pain onset with complaints of "significant muscle stretch." Left side-bending was limited to 30 degrees and right-side bending was 45 degrees. Cervical rotation was within normal limits and not overly provocative of symptoms in either direction. Upper extremity shoulder motion was within normal limits, with discomfort at end-range left shoulder flexion into this left upper trapezius region. Passive range of motion was slightly better with cervical extension to 5 degrees, cervical flexion to 45 degrees with muscle stretch end-feel, and cervical rotation within normal limits with no provocation of symptoms.

Palpation revealed no apparent deformities or tissue texture abnormalities. Manual muscle testing was deferred at the initial visit due to heightened pain with motion testing. He had significant tenderness to palpation of the left paraspinal muscles, greatest adjacent to C5-6 levels with palpable taut muscle bands of the left paraspinals, mid-portions of the left sided upper trapezius and mild tenderness at the insertion of left levator scapulae muscle. Palpation of the left first rib posteriorly elicited reproduction of the subject's pain. Passive accessory intervertebral movement assessment with anterior-to-posterior testing and up-gliding elicited the most discomfort and guarding at C5-6. The subject had difficulty positioning into cervical quadrant assessments, which were negative for radiating symptoms. The supine cervical distraction test was also negative for reduc- 


\section{Timeline of Medical \& PT Encounters}

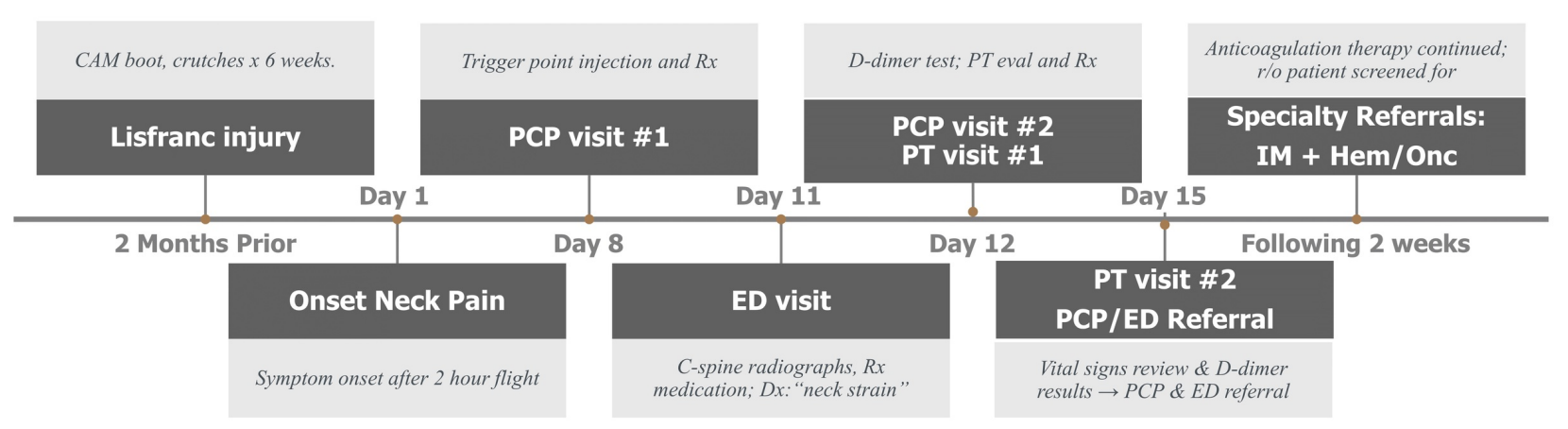

Figure 2 .

\section{Figure 1: Timeline of Medical and PT encounters}

"CAM: controlled ankle movement, PCP: primary care provider, PT: physical therapy, r/o: rule-out, IM: internal medicine, Hem/Onc: hematology oncology, Dx: diagnosis, c-spine: cervical spine, Rx: treatment/prescription, ED: emergency department

tion in symptoms. Light touch sensation and deep tendon reflexes were assessed and found to be intact and symmetrical.

The initial exam findings were relatively benign. Following a thorough review of the subject's past military medical history, the absence of significant injury or systemic disease, and an unremarkable family history, the physical therapist's initial clinical impression was that the subject's pain was most likely musculoskeletal in nature. There were no signs or symptoms suggesting significant pathology or the need for referral. ${ }^{8}$

\section{INITIAL PHYSICAL THERAPY TREATMENT}

The subject verbally consented to manual physical therapy 9 including Grade V thrust manipulation in the supine position in an anterior-to-posterior direction of the left first rib with cavitation, supine cervicothoracic junction manipulation without cavitation, prone cervicothoracic junction manipulation with cavitation, supine up-glide cervical manipulation at C5-6 without cavitation. Post-treatment cervical extension improved by 10 degrees. Due to the subject's favorable response to trigger point injection, dry needling treatment was considered an appropriate adjunct therapy. The subject provided written consent to dry needling of the left and right paraspinal muscles at levels C5-7 which provided no additional functional benefit. The subject was instructed to perform active range of motion exercises and advised to use heat or ice as needed based on preference to assist with pain. ${ }^{10}$

\section{PHYSICAL THERAPY EVALUATION \#2}

The subject returned to physical therapy for follow-up two days later (after the weekend) in visible distress and discomfort. He reported that his symptoms were slightly improved the evening after the initial physical therapy treatment and that the improvement was sustained through to the next morning, but that his pain worsened the following day. He reported feeling nauseous and described a "clawing and gripping pain” deep into his left upper trapezius and neck area, but denied numbness, tingling, or radiating symptoms into the upper extremities. With the exception of increased pain, the subject denied any changes, any potentially precipitating events, or activities that could explain his severe increase in pain. Due to the significant increase in the subject's pain, the physical therapist once more reviewed the subject's medical records, to include recent vital signs. Upon further review, it was noted that there was a subtle upward trend in the past three months of blood pressure measurements (Figure 2) following a Lisfranc injury two months prior and continued increases during the encounters for neck pain. Additionally, there was an elevated $\mathrm{D}$-dimer test result that had not yet been reviewed by the PCP. The subject's D-dimer test was valued at 261, with the normal range being reported $0-230 .{ }^{11}$ Considering the subject's intractable pain with pharmacological treatment, physical therapy intervention and especially in light of the high blood pressure readings and high D-dimer blood test, the physical therapist became more concerned that the subject's pain was not musculoskeletal in nature. ${ }^{11}$ The physical therapist initiated an immediate referral back to the subject's PCP with the suggestion of a Doppler ultrasound evaluation.

\section{OUTCOME}

The subject's PCP ordered an UE duplex ultrasound Doppler which revealed that he had several thromboses of the superficial vein network of the left upper extremity, specifically the cephalic vein and mid- and distal portions of the basilic veins. His PCP immediately referred him to the emergency department where he was screened for pulmonary embolism with a chest computed tomography (CT) which was found to be negative. ${ }^{12}$ He was also treated with an anticoagulant, Rivaroxaban (Xarelto). The subject was prescribed a therapeutic dose of Xarelto $(15 \mathrm{mg})$ to be taken orally twice daily for 21 days. After completing the first three weeks of 
anticoagulant medication, the subject's dose was decreased to $20 \mathrm{mg}$ once daily and ordered to be taken for six additional weeks. He discontinued use of Xarelto after this time. The emergency department referred the subject to specialty care in hematology/oncology to rule out more potential underlying causes of the subject's UESVTs. The hematology/ oncology specialist reported no remarkable findings, also coming to the conclusion that repetitive upper extremity use while on crutches contributed to the subject's UESVTs development. At both six-month and two-year follow-up, the subject had no recurring symptoms nor symptomatic SVTs since that time.

\section{DISCUSSION}

Despite the unlikely occurrence of UESVTs in young and healthy individuals, there are conditions which may create opportunities for thromboses to form in the upper extremity. Specifically, repetitive retroversion, hyperabduction and extension of the arm have been proposed to be mechanism by which undue stress is caused to the endothelium of the subclavian vein resulting in a coagulation cascade. ${ }^{5} \mathrm{Al}-$ though there is no literature specific to SVTs, long-haul flights (>6hr) have also been associated with a 2.3-2.8-fold increase in risk for developing venous thrombosis-related complications when compared to shorter flights. ${ }^{13,14} \mathrm{Al}-$ though it was noted in the subject's history that he had taken a short flight prior to the onset of his symptoms, there is minimal evidence that short duration flights result in DVT, SVT, or related thrombosis-related complications. ${ }^{13}$ The incidence of neck pain caused by a superficial or deep vein thrombosis (DVT) in the upper extremity is less frequent than reported cases of leg pain from a DVT. ${ }^{3}$ In light of the subject's history of Lisfranc injury followed by using bilateral axillary crutches for a prolonged period, it is plausible that this subject developed UESVTs, also described as effort thromboses, as a result of his prolonged crutch use. ${ }^{13}$ The subject was also questioned further regarding his crutch use and stated that he would often use a single crutch on his injured left-side, further contributing to repetitive stress.

While the subject in this case report did not present with any red flags at the initial evaluation, his response to treatment was concerning at follow-up. Common risk factors, clinical signs, and symptoms for SVT are not well defined, however the known risks, signs, and symptoms for DVT are presented in Table $1 .{ }^{15-18}$ While clinical signs and symptoms related specifically to UESVTs are less commonly reported in the literature, a common clinical sign of UEDVT, including $\mathrm{PE}$, is increased heart rate, ${ }^{15}$ while edema of the arm or hand may occur, it is less common. ${ }^{16}$ A review of the subject's electronic medical record (EMR) demonstrated no significant increases in heartrate or respiratory rate. The subject's EMR did show gradual, but notable increases in blood pressure beginning shortly after the onset of his LE injury several months prior, peaking at his second physical therapy appointment. Blood pressure has not been identified as a predictive clinical sign of UEDVT or UESVT and may have been reflective of the body's natural response to pain, ${ }^{19}$ nonetheless, observation of prolonged, elevated, and rising blood pressure prompted the physical therapist

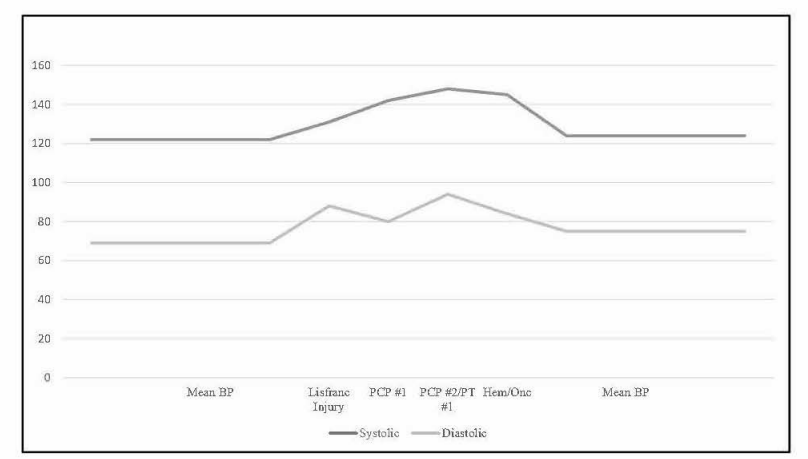

\section{Figure 2: Patient Blood Pressure Timeline}

*PCP: Primary Care Provider; PT: Physical Therapist; Hem/Onc: Hematology Oncology

to consult with the subject's PCP. Other findings associated with an increased risk of thrombosis include having blood type $\mathrm{AB}$ or factor $\mathrm{V}$ gene mutation. ${ }^{17,20}$ The subject described in this case report had type $O$ positive blood, and his factor V gene mutation was not reported. Physical therapists suspecting a thrombosis in their subject should include blood type and factor $\mathrm{V}$ gene mutation results in their consideration of risk for thrombosis. Individuals with blood clotting disorders such as antithrombin III, protein S, or protein $\mathrm{C}$ deficiencies may also have an increased risk for developing thrombosis. ${ }^{16}$ Finally, medications may contribute to increased risk of thrombosis, ${ }^{21}$ such as oral contraceptives, antipsychotics, and alcohol. The subject described in this case report did not have a history of medication or substance misuse which might contribute to an increased risk or susceptibility to developing an UESVT. Objective findings of elevated blood pressure and a borderline high D-Dimer triggered an immediate referral to a physician for additional testing. ${ }^{11}$ A physical therapist acting in a direct access role has sufficient training to screen and identify more sinister diagnoses, like the one presenting in this case. ${ }^{22,23}$

An SVT is not inherently life-threatening on its own, but could potentially lead to a more concerning DVT or pulmonary embolism (PE). 3,5,24 Subjects who are typically diagnosed with UESVTs are older and have multiple comorbidities $^{25}$, so it was out of the ordinary to find one in this younger, healthy individual. The military setting is unique in that healthcare providers have immediate access to all health and medical records for a subject. In civilian outpatient populations, this subject might have not been referred as quickly to the appropriate provider without history of vital records. Physical therapists operating in environments without access to vital records should consider obtaining vital signs, including blood pressure measurement, on all of their subjects.

UESVTs are more commonly seen in older subjects ( $>65$ years), with multiple comorbidities demonstrating clinical signs of increased heart rate, indurated cord, upper extremity discoloration, and/or arm and hand edema. Notably, these clinical signs are only seen in smaller percentages of the population and are inconsistent across individuals. ${ }^{16}$ Other findings which might alert a physical therapist to 
Table 1: Predictors of SVT, DVT, \& PE Compared to Case Report Patient Characteristics

\begin{tabular}{|c|c|c|}
\hline Variable & Characteristics of Patients with SVT, DVT, \& PE* & Patient characteristics \\
\hline \multirow[t]{18}{*}{ Risk Factors (\%) } & 62 years (range: $48-73)^{1}$ & 27 years \\
\hline & Female (66) & Male \\
\hline & Confinement to bed (5) & No \\
\hline & Recent travel (7) & Yes \\
\hline & Congestive heart failure or respiratory insufficiency (5) & No \\
\hline & Acute infectious disease (4) & No \\
\hline & Pregnancy or post-partum period $<6$ weeks (5) & $\mathrm{n} / \mathrm{a}$ \\
\hline & Personal history of VTE (43) & No \\
\hline & Family history of VTE (26) & No \\
\hline & Cancer (8) & No \\
\hline & Active cancer (4) & No \\
\hline & Previous cancer (4) & No \\
\hline & SVT in a non-varicose vein (22) & Yes \\
\hline & Chronic reduced mobility (4) & No \\
\hline & Biological thrombophilia (5) & No \\
\hline & Hormonal contraception (5) & $\mathrm{n} / \mathrm{a}$ \\
\hline & Hormone replacement therapy (2) & No \\
\hline & Obesity (BMI >30 kg/m²) & No $(\mathrm{BMI}=23.7)$ \\
\hline \multirow[t]{2}{*}{ Symptoms } & Vague shoulder or neck discomfort ${ }^{3}$ & $\begin{array}{l}\text { Severe neck and upper } \\
\text { shoulder pain** }\end{array}$ \\
\hline & Arm or hand edema ${ }^{3}$ & Not present \\
\hline \multirow[t]{7}{*}{ Signs } & Heart rate $75-94$ beats $/ \mathrm{min}^{2}$ & $53-71$ beats $/ \mathrm{min}$ \\
\hline & Supraclavicular fullness ${ }^{2}$ & Not present \\
\hline & Palpable cord ${ }^{2}$ & Not present \\
\hline & Extremity cyanosis ${ }^{2}$ & Not present \\
\hline & Dilated cutaneous veins ${ }^{2}$ & Not present \\
\hline & Jugular venous distention ${ }^{2}$ & Not present \\
\hline & Unable to access central venous catheter ${ }^{2}$ & $\mathrm{n} / \mathrm{a}$ \\
\hline Blood type & $\mathrm{AB}^{4}$ & $\mathrm{O}+$ \\
\hline \multicolumn{3}{|l|}{ Lab values } \\
\hline Factor V Gene Mutation & Reported in up to $11 \%$ of patients diagnosed UEDVTs ${ }^{3}$ & Not reported \\
\hline Antithrombin III deficiency & Reported in $2-4 \%$ of patients diagnosed with UEDVTs 3 & $\begin{array}{l}\text { Within range } 108 \\
\text { (reference range } 75-135 \text { ) }\end{array}$ \\
\hline Protein S deficiency & Present in $\sim 4 \%$ of patients diagnosis with UEDVTs ${ }^{3}$ & $\begin{array}{l}\text { High } 161 \text { (reference range } \\
63-140)^{* *}\end{array}$ \\
\hline Protein C deficiency & Present in $4-7 \%$ of patients diagnosed with UEDVTs ${ }^{3}$ & $\begin{array}{l}\text { Within range } 109 \\
\text { (reference range 73-180) }\end{array}$ \\
\hline
\end{tabular}

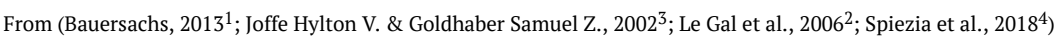

"Literature is limited on signs and symptoms related to SVT; signs \& symptoms of DVT, PE included here.

*** Indicates potential marker for SVT, DVT, or PE evident in patient.

BMI: body mass index; SVT: superficial vein thrombosis; VTE: venous thromboembolism

possible UESVTs include the subject having an $\mathrm{AB}$ blood type, having factor $\mathrm{V}$ gene mutations, or being on thrombosis-inducing medication such as oral contraceptives. Finally, in populations where UESVT might be less commonly suspected, physical therapists might consider PagetSchroetter Syndrome (effort thrombosis) or Thoracic Outlet
Syndrome (TOS). ${ }^{4,5}$ In these rare cases, the presence of repetitive upper extremity movements cause microtrauma to the endothelium creating thromboses described in this subject due to overuse of an axillary crutch on the affected side. While the incidence of UESVTs is quite low, a thorough subjective and objective physical therapy examination, with 
reflective consideration of subject response to treatment, are likely to lead to appropriate referral.

\section{CONCLUSION}

Systems review with vital signs assessment (i.e., heart rate, blood pressure, respiratory rate, and temperature) provide valuable objective information related to patient status, potentially revealing undiagnosed medical conditions, and giving a snapshot of a patient's general health and well-being. Physical therapists should collect vitals for each patient at their initial evaluation regardless of past medical history; subsequent readings can be used as a comparison and possible indication for physician referral. The subject described in this case report was younger and otherwise healthier than the typical patient who might present with an UESVT. Aside from consulting the ultrasound Doppler and CT findings, the only indication of thrombosis was a steady rise in blood pressure across several healthcare visits. Performing a thorough systems review with vital signs assessment adds minimal time and should be considered standard practice for patient encounters.

\section{CONFLICTS OF INTEREST}

The authors have no conflicts of interest to disclose.

"The subject described in this case report was informed that the data concerning the case would be submitted for publication and subject confidentiality has been protected according to the U.S. Health Insurance Portability and Accountability Act."

Submitted: July 16, 2020 CDT, Accepted: December 15, 2020 CDT 


\section{REFERENCES}

1. Hoy D, March L, Woolf A, et al. The global burden of neck pain: estimates from the global burden of disease 2010 study. Ann Rheum Dis.

2014;73(7):1309-1315. doi:10.1136/annrheumdis-201 $\underline{3-204431}$

2. Kim R, Wiest C, Clark K, Cook C, Horn M. Identifying risk factors for first-episode neck pain: A systematic review. Musculoskelet Sci Pract. 2018;33:77-83. doi:10.1016/j.msksp.2017.11.007

3. Ageno W, Haas S, Weitz JI, et al. Upper extremity DVT versus lower extremity DVT: perspectives from the GARFIELD-VTE registry. Thromb Haemost. 2019;119(8):1365-1372. doi:10.1055/s-0039-1688828

4. Ploton G, Pistorius M-A, Raimbeau A, et al. A STROBE cohort study of 755 deep and superficial upper-extremity vein thrombosis. Medicine. 2020;99(6). doi:10.1097/MD.0000000000018996

5. Alla VM, Natarajan N, Kaushik M, Warrier R, Nair CK. Paget-Schroetter Syndrome: Review of pathogenesis and treatment of effort thrombosis. West J Emerg Med. 2010;11(4):358-362.

6. Nasr H, Scriven JM. Superficial thrombophlebitis (superficial venous thrombosis). BMJ. 2015;350(jun22 6):h2039-h2039. doi:10.1136/bmj.h2039

7. Williamson A, Hoggart B. Pain: a review of three commonly used pain rating scales. J Clin Nurs. 2005;14(7):798-804.

8. Bier JD, Scholten-Peeters WGM, Staal JB, et al. Clinical practice guideline for physical therapy assessment and treatment in subjects with nonspecific neck pain. Phys Ther. 2018;98(3):162-171.

9. Masaracchio M, Cleland JA, Hellman M, Hagins M. Short-term combined effects of thoracic spine thrust manipulation and cervical spine nonthrust manipulation in individuals with mechanical neck pain: a randomized clinical trial. J Orthop Sports Phys Ther. 2013;43(3):118-127. doi:10.2519/jospt.2013.422 1

10. Childs JD, Cleland JA, Elliott JM, et al. Neck pain: Clinical practice guidelines linked to the International Classification of Functioning, Disability, and Health from the Orthopedic Section of the American Physical Therapy Association. J Orthop Sports Phys Ther. 2008;38(9):A1-A34. doi:10.2519/jos pt.2008.0303
11. Sartori M, Migliaccio L, Favaretto E, et al. D-dimer for the diagnosis of upper extremity deep and superficial venous thrombosis. Thromb Res. 2015;135(4):673-678. doi:10.1016/i.thromres.2015.0 $\underline{2.007}$

12. Ellis MH, Fajer S. A current approach to superficial vein thrombosis. Eur J Haematol. 2013;90(2):85-88. doi:10.1111/ejh.12044

13. Gavish I, Brenner B. Air travel and the risk of thromboembolism. Intern Emerg Med. 2011;6(2):113-116. doi:10.1007/s11739-010-0474-6

14. Onida S, Davies AH. Long-haul travel venous thromboembolism - an update. Phlebolymphology. 2020;27(1):11-16.

15. Le Gal G, Righini M, Roy P-M, et al. Prediction of pulmonary embolism in the emergency department: The revised geneva score. Ann Intern Med.

2006;144(3):165-171. doi:10.7326/0003-4819-144-3-2 00602070-00004

16. Joffe Hylton V., Goldhaber Samuel Z. Upperextremity deep vein thrombosis. Circulation. 2002;106(14):1874-1880. doi:10.1161/01.CIR.0000031 705.57473.1C

17. Spiezia L, Campello E, Valle FD, Simion C, Colpo A, Simioni P. ABO blood group and the risk of postthrombotic syndrome. Ann Hematol. 2018;97(6):1057-1060. doi:10.1007/s00277-018-325 $\underline{5-3}$

18. Bauersachs RM. Diagnosis and treatment of superficial vein thrombosis. Hamostaseologie. 2013;33(3):232-240. doi:10.5482/hamo-13-04-0027

19. Tousignant-Laflamme Y, Rainville P, Marchand S. Establishing a link between heart rate and pain in healthy subjects: A gender effect. J Pain. 2005;6(6):341-347. doi:10.1016/j.jpain.2005.01.351

20. Martinelli I, Cattaneo M, Taioli E, Stefano VD, Chiusolo P, Mannucci PM. Genetic risk factors for superficial vein thrombosis. Thromb Haemost. 1999;82(10):1215-1217. doi:10.1055/s-0037-1614362

21. Ramot Y, Nyska A, Spectre G. Drug-induced thrombosis: An update. Drug Saf. 2013;36(8):585-603. doi:10.1007/s40264-013-0054-6 
22. Rhon DI, Gill N, Teyhen D, Scherer M, Goffar S. Clinician perception of the impact of deployed physical therapists as physician extenders in a combat environment. Mil Med. 2010;175(5):305-312. doi:10.7205/milmed-d-09-00099

23. Ross MD, Childs JD, Middel C, et al. Physical therapist vs. family practitioner knowledge of simple low back pain management in the U.S. Air Force. Mil Med. 2014;179(2):162-168. doi:10.7205/MILMED-D-1 $\underline{\text { 3-00099 }}$
24. Leon L, Giannoukas AD, Dodd D, Chan P, Labropoulos N. Clinical significance of superficial vein thrombosis. Eur J Vasc Endovasc Surg. 2005;29(1):10-17. doi:10.1016/j.ejvs.2004.09.021

25. Litzendorf ME, Satiani B. Superficial venous thrombosis: disease progression and evolving treatment approaches. Vasc Health Risk Manag. 2011;7:569-575. doi:10.2147/VHRM.S15562 